\title{
Liquid Isolation to Optimize Ultrasonic Display of Cervical Lymphatic Vessels
}

\author{
Jianquan Zhang, $M D^{a, b, *}$, Hongqiong Chen, $M D^{a}$, Lei Yan, $M D^{c}$, Jie Cheng, $M^{a}$ \\ ${ }^{a}$ Department of Ultrasound, Super-minimally Invasive Surgery, Shanghai International Medical Center, Shanghai, China; ${ }^{b}$ Department \\ of Medical Ultrasound, Shanghai Changzheng Hospital, Shanghai, China; ${ }^{c}$ Department of Medical Ultrasound, The 904th Hospital of \\ PLA, Suzhou City, China
}

Received May 09, 2020; revision received June 04, 2020; accepted June 08, 2020

\begin{abstract}
Lymphatic vessels are the only pathway for lymphatic fluid to flow into venous bloodstream, and they are also one of the main channels for migration and metastasis of malignant tumor cells. High-frequency ultrasound imaging has been used popularly as first choice for examination and preliminary assessment of cervical lymph nodes. However, its capacity of displaying lymphatic vessels is still insufficient up to date. Recently, liquid isolation has been increasingly used in ultrasoundguided thermal ablation treatment of thyroid nodules, parathyroid nodules, or cervical lymph nodes with malignant metastasis, playing a key role of protecting adjacent surrounding structures. In this paper, the role of liquid isolation in optimizing the ultrasonic display of cervical lymphatic vessels was presented and further likely novel technique based on this case was outlook as well.
\end{abstract}

Key words: Cervical lymph node; Cervical lymphatic vessel; Ultrasonic display; Liquid isolation; Fine needle aspiration

Advanced Ultrasound in Diagnosis and Therapy 2020;03:251-254

DOI: 10.37015/AUDT.2020.200044

$\mathrm{L}$ ymphatic vessels are the only pathway for lymphatic fluid to flow into venous bloodstream, and they are also one of the main channels for the migration and metastasis of malignant tumor cells [1]. Among the various neck malignancies, differentiated thyroid carcinomas (DTCs) are characterized by metastases to regional lymph nodes via lymphatic vessels $[2,3]$. Although high-frequency ultrasound imaging has become the first choice for examination and preliminary assessment of cervical lymph nodes, obviously its capacity of displaying the lymphatic vessels is still insufficient up to date [4]. Attempts have been made to enhance the ultrasonographic display of lymphatic vessels by interstitial injection of contrast agents closely surrounding thyroid carcinoma [5-7], but definitive results have not been obtained due to a deal of limits. If we can improve the display of lymphatic vessels by commonly used two-dimensional ultrasound imaging, it may be helpful to the relevant research and application in this field.
In this paper, we report a case of papillary thyroid carcinoma in which the sonographic features of lymphatic vessels were revealed by using liquid isolation technique during the procedure of ultrasound-guided fine-needle aspiration (FNA) on his left cervical lymph node for cytopathology. In addition, we outlook the likely technological innovation and theoretical progress about lymphatic investigation herein.

\section{Case Report}

\section{Clinical Features}

A 22-year-old man was transferred to our hospital due to an undetermined papillary thyroid carcinoma (PTC)like nodule incidentally disclosed during a massive ultrasound screening examination. He denied any complaint of clear clinical manifestations and eagerly sought for ultrasound-guided fine needle aspiration cytopathology (FNAC) to make a definite diagnosis of his left thyroid nodule.

\footnotetext{
* Corresponding author: Department of Ultrasound in Medicine, Changzheng Hospital, 415 Fengyang Road, Shanghai, China e-mail: thyroid_ablation@vip.sina.com unrestricted use, distribution and reproduction in any medium provided that the original work is properly attributed.
} 


\section{Neck Ultrasonographic Findings}

Prior to fine needle aspiration (FNA), ultrasound examination (with using the ultrasonic apparatus of Preius, HITACHI, Japan) showed that the left thyroid gland was slightly enlarged, and a moderately low echogenic nodule were seen in the middle-upper part of the gland, with clear boundary, scattered strong echoes in the interior, and no obvious acoustic shadow in the behind area. Color Doppler flow imaging (CDFI) showed abundant blood flow signals in the nodules (Fig. 1). No clear malignant sonographic features were found both in the rest of his thyroid glands and in the bilateral cervical lymph nodes, except one lymph node in the left cervical region III was slightly larger than the other lymph nodes in the same region, and a foci of calcification was suspected sited within the nodal cortex (Fig. 2). In fear of potential lymphatic metastasis, the patient requested that the lymph node be biopsied with FNA too.
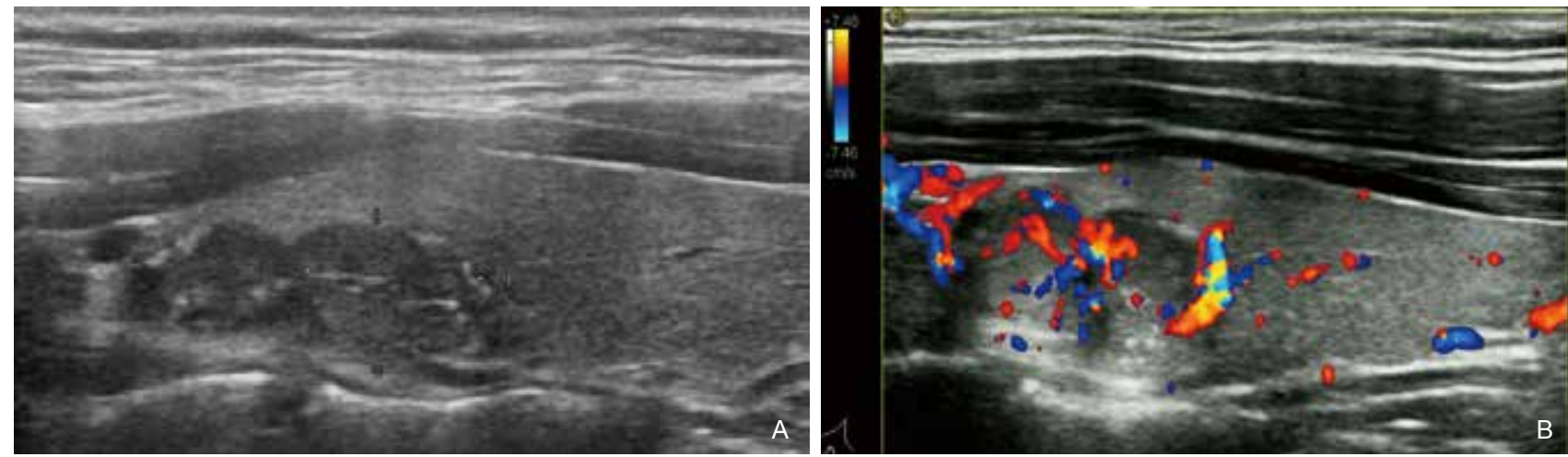

Figure 1 Ultrasonograms of a papillary carcinoma-suspected left thyroid nodule. (A) A moderately-defined hypoechoic nodule in the middle-upper part of the thyroid with scattered strong echoes and slight posterior enhanced echoes. (B) Display of color signals within the partial parenchyma of the nodule on CDFI mode.
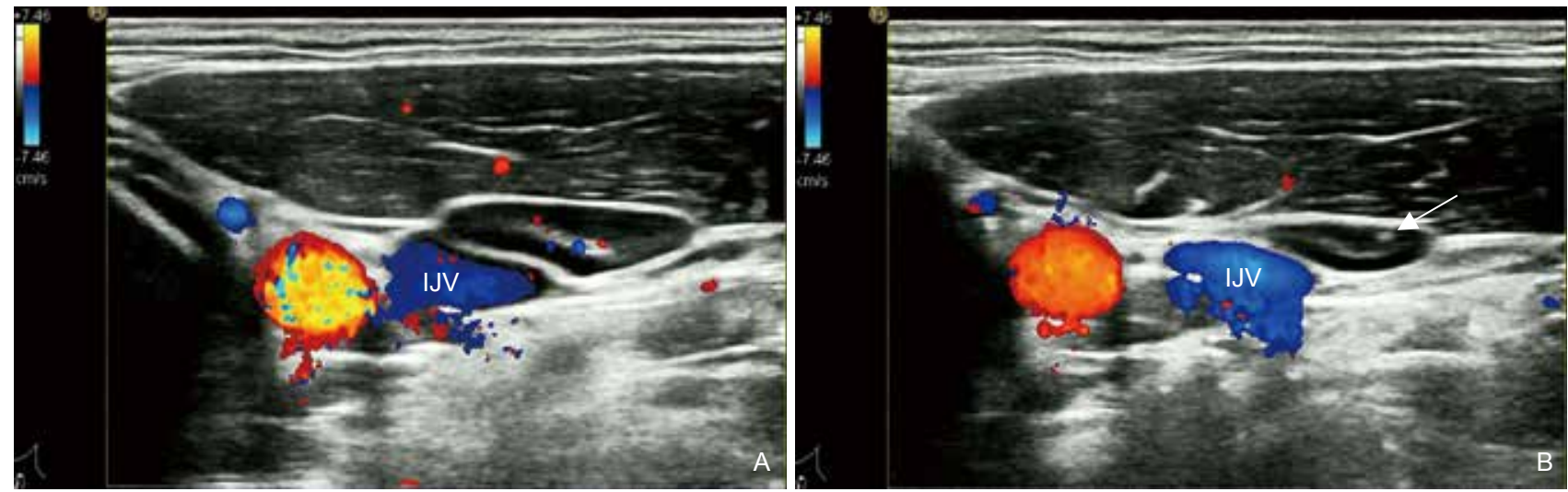

Figure 2 Ultrasonograms of cervical lymph node at the region III. (A) The lymph node was tightly wrapped with smooth and uniform hyperechoic capsule, along with homogeneously hypoechoic cortex and clearly identified central hyperechoes of the hilum. (B) A hyperechoic foci of calcification was suspected near the upper pole of the node (arrow indicating). IJV, internal jugular vein.

\section{Ultrasound-guided Percutaneous FNA}

Under the guidance of high-frequency ultrasound (with using the ultrasound apparatus of Resona 7S, Mindray, China), 1\% lidocaine solution was used to anesthetize and analgesize the sterilized skin puncture points and subcutaneous puncture paths. FNA was carried out consecutively on the left thyroid nodule and the left cervical lymph node at region III, by using a fine needle (PA, Italy) in length of $8 \mathrm{~cm}$ and outer diameter of 23 Gauge. Since the lymph node was attached to the anterior wall of the internal jugular vein, fluid isolation was performed between them to improve the convenience and safety of the FNA operation.

\section{Emerging of suspected lymphatic ducts during fluid isolation}

Before fluid isolation, the capsule of lymph node was clear and smooth on the ultrasound imaging, and no tubular structure was seen attached to it. During fluid isolation, as the isolation zone was widened, several slender tubular structures were seen in the isolation fluid and they all had one end being connected to the capsule 
of the lymph node. By further increasing injection of isolating fluid, the tubular structures were seen extending but never separating from the lymph nodes, while the fascia around the lymph nodes was in a strip-like manner separating from the lymph nodes (Fig. 3).
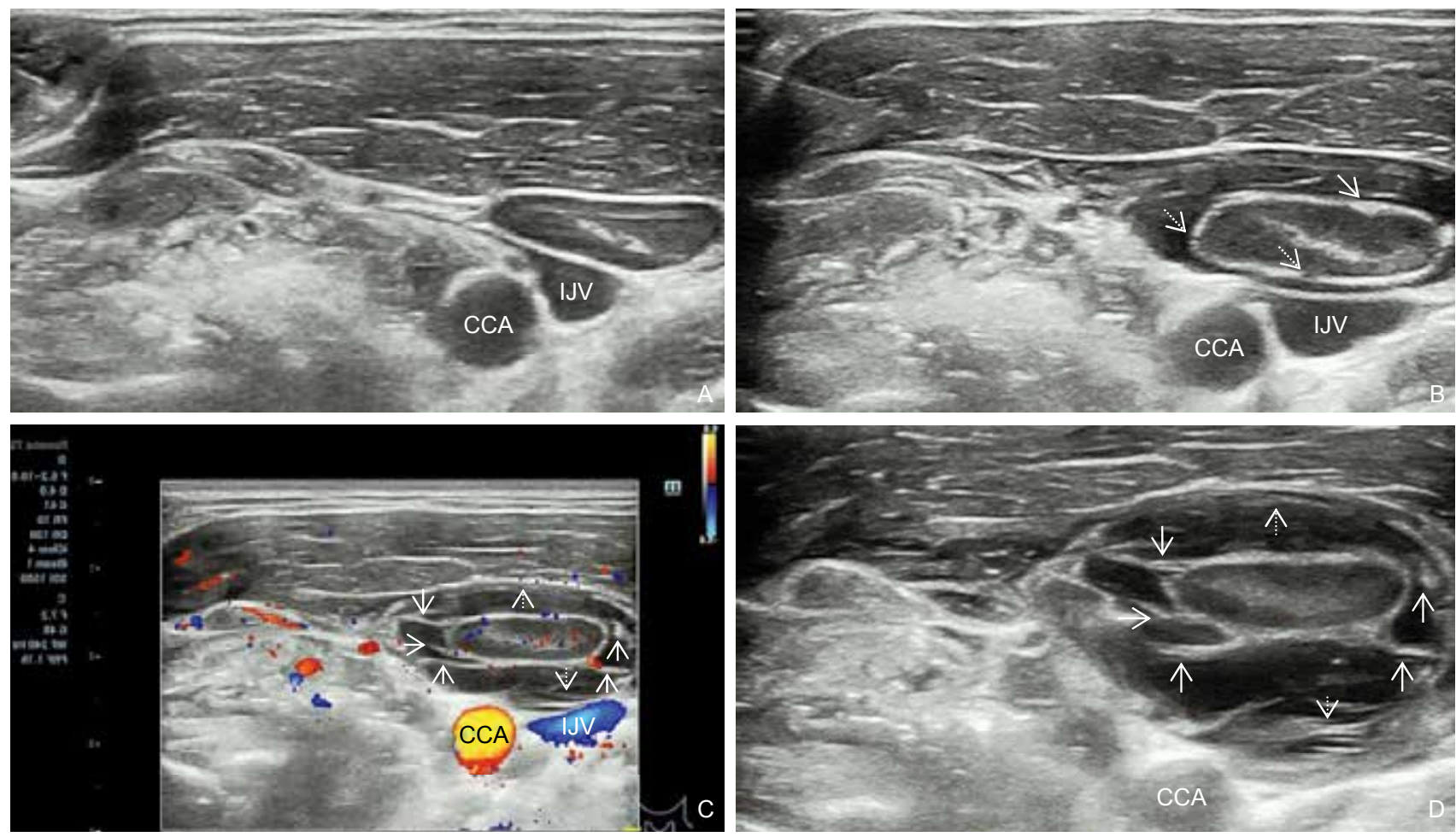

Figure 3 Ultrasonograms of the lymph node and the suspected lymphatic vessels. (A) In the left cervical region III a lymph node under the natural state shows a clear and continuous hyperechoic capsule and homogeneous hypoechoic cortex. It fits fairly close to the adjacent structures without any visible interspaces, and moreover no tubular structure is visible in connection to it. (B) After injection of $5 \mathrm{ml}$ of sterilized saline into the latent interspaces, the lymph node got separated dynamically from the close surroundings and immersed in the saline. Meanwhile, the nodal capsule was found to have focal thickening (solid open arrow) and fine cracks (dashed open arrows). (C) Continued to increase injection of 10ml of saline, the isolation zone got widened, at least 5 slender tube or cord-like strong echogenics (solid open arrows) appeared in the isolation fluid, with one end connected to the capsule of the lymph node. Some strip-like parts (dashed open arrows) were visible in the peripheral area of the isolation zone, which were not connected with the lymph node capsule. (D) With much more injection of saline, the isolation zone turned much wider, and the tubular feature (solid open arrows) turned more clearly, and their junction with the lymph node was more definite. The strip-like parts (dashed open arrows) in the peripheral area of the isolation zone were further away from the lymph node. IJV, internal jugular vein; CCA, common carotid artery.

\section{FNAC of left thyroid nodule and left cervical lymph node at region III}

FNAC disclosed the entity of papillary thyroid carcinoma in the extracts from the left thyroid nodule (Fig. 4), while revealing only numerous lymphocytes without evidence indicating any malignancy in the specimen taken from the left cervical lymph node (Fig. 5).

\section{Discussion}

It is a common consensus that lymphatic vessels are invisible on conventional ultrasound imaging modes, including two-dimensional gray-scale imaging, CDFI, and intravenous contrast-enhanced ultrasonography (CEUS). However, our preliminary study has revealed that fluid isolation technique is capable of optimizing the ultrasound display and identification of lymphatic vessels.

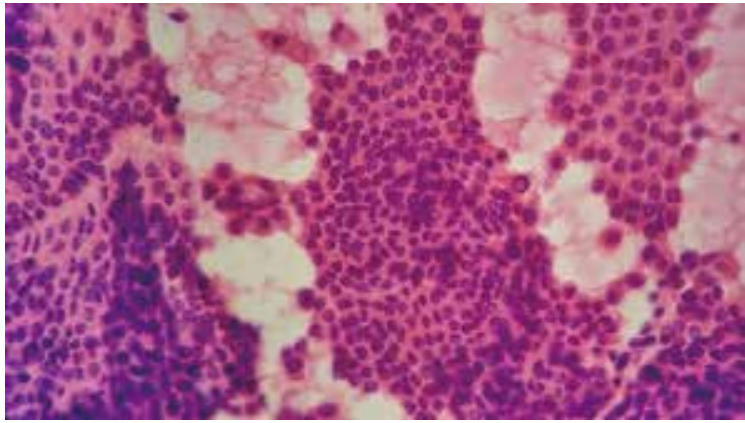

Figure 4 FNAC of the left thyroid nodule. Microscopically, the thyroid follicular epitheliums were closely packed, overlapped, and arranged in papillary shape, with distinctive nuclear groove and pseudo-inclusion bodies visible (original magnification $\times 400$ ).

Lymphatic vessels are slender and widely distributed in the neck area. Usually five or six afferent lymphatic tubes converge to a lymph node and one or two efferent 
lymphatic tubes leave the lymph node. Therefore, taking lymph nodes as the start-point makes it more sensible and feasible to explore the methods and manifestations of ultrasonic display of the afferent and efferent lymphatics.
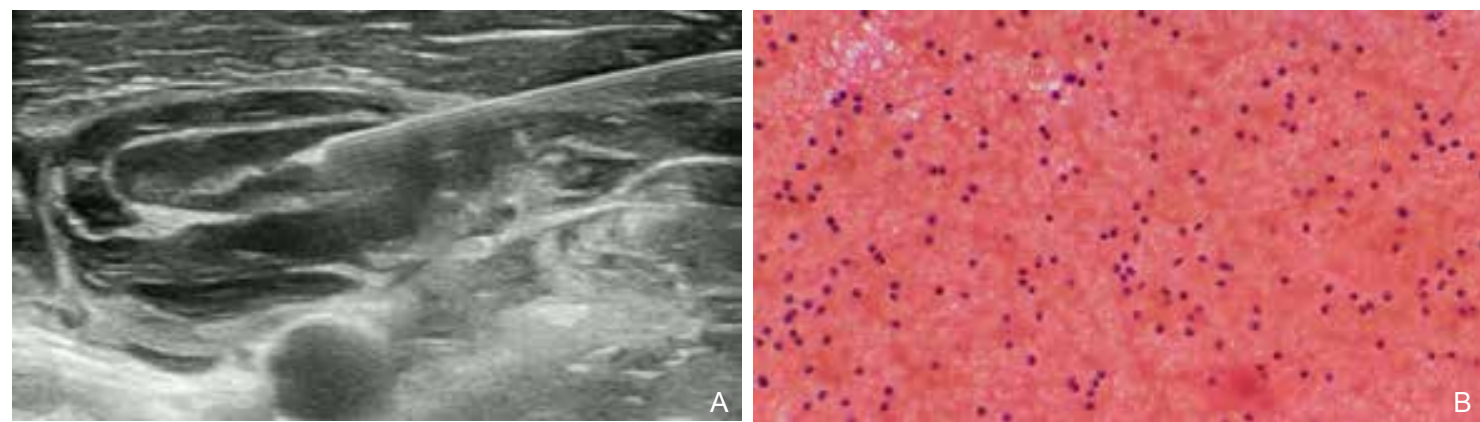

Figure 5 FNA and FNAC of the left cervical lymph node. (A) FNA was operated on the lymph node in fashion of multiple passages combined with targeted aspiration of the suspected foci of calcification; (B) Microscopic examination demonstrated abundant of normal lymphocytes and erythrocytes, but no heterocyst (original magnification $\times 400$ )

We believe that lymphatic vessels are invisible on conventional ultrasound imaging is probably because of the insufficient acoustic impedances between the vessels and their adjacent structures. However, they get visible on conventional ultrasound imaging while they are immersed in isolation fluid in the way stated in this paper. One reason for this result is believed to be the sufficient acoustic impedance between the lymphatic ducts and isolation liquid. Another reason for this finding is lymphatic ducts separate away from the adjacent tissue under the tension force of isolation liquid. In physiologic situation, the lymphatic vessels are tightly attached but not adhesive to their surrounding tissues because they are of good conformal ability due to their soft tube walls and tiny tube diameter. Liquid isolation makes the potential peri-lymphatic space get widened, which is an artificial situation under which the lymphatic ducts go extended and produce significant acoustic reflection. Therefore, we can conclude that liquid isolation method helps to optimize the identification of lymphatic vessels on conventional ultrasound imaging.

Although the results in this paper are only a preliminary finding, the potential value of ultrasound display of lymphatic vessels is expectable and encouraging. Display and identification of lymphatic duct by conventional ultrasound imaging is meaningful to promote ultrasound-guide puncture of lymphatic duct, which has been rarely reported in literatures.

With the help of this novel puncture technique, translymphatic injection of ultrasound contrast agent could be performed to realize the innovation and expansion of contrast-enhanced ultrasonography on mapping the lymphatic flow in head and neck region. In addition, this novel puncture technique could further make it possible to search for tumor cells by extracting lymphatic fluid, which is meaningful to provide a strong support for the classic theory of lymphatic metastasis of malignant tumor cells.

\section{Acknowledgement}

This work was supported by The National Natural Science Foundation of China (81171436).

\section{Conflict of Interest}

The authors report no conflict of interest in this work.

\section{References}

[1] Zhu J, Lin S, Leow CH, Rowland EM, Riemer K, Harput S, et al High Frame Rate Contrast-Enhanced Ultrasound Imaging for Slow Lymphatic Flow: Influence of Ultrasound Pressure and Flow Rate on Bubble Disruption and Image Persistence. Ultrasound Med Biol 2019; 45: 2456-70.

[2] Garcia EA, Simões K, Wakamatsu A, Ressio RA, Alves VA, Longatto-Filho A, et al. Lymphatic vessel density and VEGF-C expression are significantly different among benign and malignant thyroid lesions. Endocr Pathol 2010; 21: 101-7.

[3] Mai KT, Truong LD, Ball CG, Olberg B, Lai CK, Purgina B. Lymphatic endothelial cancerization in papillary thyroid carcinoma: hidden evidence of lymphatic invasion. Pathol Int 2015; 65: 220-30.

[4] Hayashi A, Visconti G, Yamamoto T, Giacalone G, Hayashi N, Handa $\mathrm{M}$, et al. Intraoperative imaging of lymphatic vessel using ultra highfrequency ultrasound. J Plast Reconstr Aesthet Surg 2018; 71: 778-80.

[5] Gelb HR, Freeman LJ, Rohleder JJ, Snyder PW. Feasibility of contrast-enhanced ultrasound-guided biopsy of sentinel lymph nodes in dogs. Vet Radiol Ultrasound 2010; 51: 628-33.

[6] Nielsen Moody A, Bull J, Culpan AM, Munyombwe T, Sharma N, Whitaker M, et al. Preoperative sentinel lymph node identification, biopsy and localisation using contrast enhanced ultrasound (CEUS) in patients with breast cancer: a systematic review and meta-analysis. Clin Radiol 2017; 72: 959-71.

[7] Wakisaka N, Endo K, Kitazawa T, Shimode Y, Kato K, MoriyamaKita $\mathrm{M}$, et al. Detection of sentinel lymph node using contrastenhanced agent, Sonazoid( ${ }^{\mathrm{TM}}$ ), and evaluation of its metastasis with superb microvascular imaging in oral and oropharyngeal cancers: a preliminary clinical study. Acta Otolaryngol 2019; 139: 94-99. 